\title{
The Expression and Significance of STAT6, VEGF and MVD in Nasal Polyps
}

\author{
Qingsong Cao ${ }^{1,}$, , Jie Zhang ${ }^{2}$ \\ ${ }^{1}$ Department of Otorhinolaryngology, The First Affiliated Hospital, Jinan University, Guangzhou, China \\ ${ }^{2}$ First Class Ward, The First Affiliated Hospital, Jinan University, Guangzhou, China
}

Email address:

caoqingsong@jnu.edu.cn (Qingsong Cao)

${ }^{*}$ Corresponding author

\section{To cite this article:}

Qingsong Cao, Jie Zhang. The Expression and Significance of STAT6, VEGF and MVD in Nasal Polyps. Cancer Research Journal. Vol. 6, No. 2, 2018, pp. 47-50. doi: 10.11648/j.crj.20180602.12

Received: February 15, 2018; Accepted: March 1, 2018; Published: March 22, 2018

\begin{abstract}
The aim of the current study was to evaluate the expression levels of STAT6, VEGF and microvessel density (MVD) score in nasal polyp tissues and to explore the underlying relationships during the pathogenesis of nasal polyps. 40 cases with surgical removal of nasal polyps were included, comparing with control group of 10 inferior turbinate mucosal tissues resected from nasal septum deviation correction surgery, from the same time period. By immunohistochemistry, the protein expression levels of STAT6, VEGF and MVD were determined. The results showed that the positive expression rate of STAT6 in mucosal epithelium and glands of nasal polyp tissues was 70\%, which was significantly higher than that in control group $(P<0.01)$. The positive expression percentage of VEGF was $75 \%$, which also higher than that in control group $(P<$ 0.01 ). The MVD values in nasal polyp group was $22.67 \pm 7.54$, while $7.04 \pm 2.63$ in control group. The expression of STAT6 was closely related with the expression of VEGF and MVD, increasing with the level of VEGF and MVD. In general, the expression levels of STAT6, VEGF and MVD in nasal polyp group were increased and themselves were positively correlated, indicating the significant importance in the development of nasal polyp.
\end{abstract}

Keywords: Nasal Polyp, STAT6, VEGF, MVD, Nasal Turbinate Tissues

\section{Introduction}

The pathology of nasal polyp is a process of imbalance between cell proliferation and apoptosis, which involves in many cytokines [1]. Regulation the proliferation and apoptosis of epithelial cells is a new way to control the chronic inflammatory reaction process [2]. Signal transducer and activator of transcription (STAT) proteins are cytoplasmic proteins which can be activated to translocate to nuclei to bind DNA. Thus, STAT proteins show both signal transduction and transcriptional activity. Recently, STAT6, one isoform of STAT proteins, not only plays an important role in inflammatory regulation, but also in cell proliferation, apoptosis and cancer [3-5]. Vascular endothelial growth factor(VEGF), also called vessel pass factor (VPF), is a glycosylated secreted polypeptide factor, and is the strongest, most specific and important angiogenic factor [6]. By binding to its receptors in vascular endothelial cells, VEGF would induce cell proliferation, capillary formation and increase vascular permeability. Human leukocyte antigen CD34 is a specific marker for vascular endothelial cells, expressed in hematopoietic progenitor cells, small vascular endothelial cells, primitive mesenchymal cells and various tumor stromal small vessels $[7,8]$. CD34 antibody is usually applied for the detection of microvessel density (MVD). The expression of VEGF and CD34 have been reported in nasal polyp, however, the role of MVD and the relationship of STAT6 and VEGF in the development of nasal polyp remain unknown. By immunohistochemistry, we detected the expression of STAT6 and VEGF, the value of MVD in nasal polyp tissues and analyzed the relationship between them.

\section{Methods}

\subsection{Clinical Tissue Collection}

Nasal polyp tissues (experimental group) were collected 
form 40 patients in the Department of Otorhinolaryngology, The First Affiliated Hospital of Jinan University from Feb to Dec 2015. 26 cases of male, 24 females; average age 38.7, range $17-66$ years old. Inferior turbinate mucosal tissues (control group) were collected from 10 cases under nasal septum deviation correction surgery within the same time period; 6 males and 4 females; average age 35.3, range $18-$ 56. All the tissues were split into 2 pieces, one for $\mathrm{HE}$ staining for the detection of nasal polyp; the other was use for immunohistochemistry staining.

\subsection{Immunohistochemistry}

Briefly, tissue sections were treated with hydrogen peroxide to inactivate endogenous peroxidases. Antigen retrieval was performed in a microwave in $10 \mathrm{mM}$ citrate buffer at $\mathrm{pH}$ 6.0. Sections were fixed with paraformaldehyde followed by permeabilization and blocking. Sections were then incubated with antibodies (VEGF, 1:100; CD34, 1:25; STAT6, 1:50; all were purchased from Abcam) overnight at $4^{\circ} \mathrm{C}$, and a secondary antibody was used to detect protein expression, under a microscope (CH-30, Olympus). The images were analyzed by BI2000 software.

\subsection{Statistical Analysis}

All experiments were repeated at least three times, and the results are presented as the mean \pm SD. Analyses of significance were performed using Student's t-tests or oneway ANOVAs, followed by Bonferroni corrections. SPSS 20.0 software was used for statistical analyses. $\mathrm{P}<0.05$ was considered statistically significant.

\section{Results}

The staining results showed that STAT6 was expressed in nasal polyp glands and mucosal epithelium, but also in interstitial and infiltration of inflammatory cells. Most positive signals were distributed in nuclei, few in cytoplasmic. The positive staining rate of STAT6 was $70 \%$, with no signal in inferior turbinate tissues (Figure 1). The expression of STAT6 was significantly higher than that in inferior turbinate tissue $\left(\chi^{2}=11.8, P<0.01\right)$.
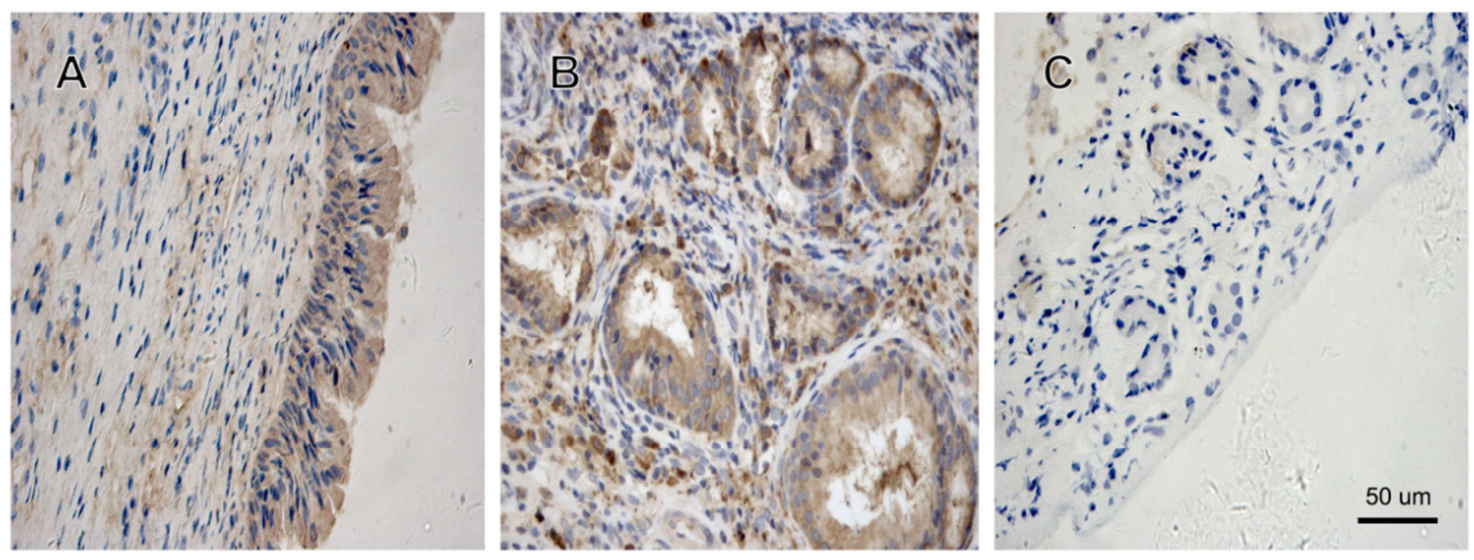

Figure 1. The expression of STAT6. (A) STAT6 in mucosal epithelium of nasal polyps. (B) STAT6 in glandular epithelium of nasal polyps. (C) STAT6 in inferior turbinate tissue (low expression).

VEGF mainly expressed in endothelium, vascular endothelial cells, glands, mucosal cells and interstitial infiltration of inflammatory cells of nasal polyp tissues. Most positive staining signals were shown in nuclei. The positive rate of VEGF was $75 \%$, with weak staining in inferior turbinate tissue (Figure 2). The expression of VEGF was significantly higher than that in inferior turbinate tissue $\left(\chi^{2}=\right.$ $13.9, P<0.01)$.
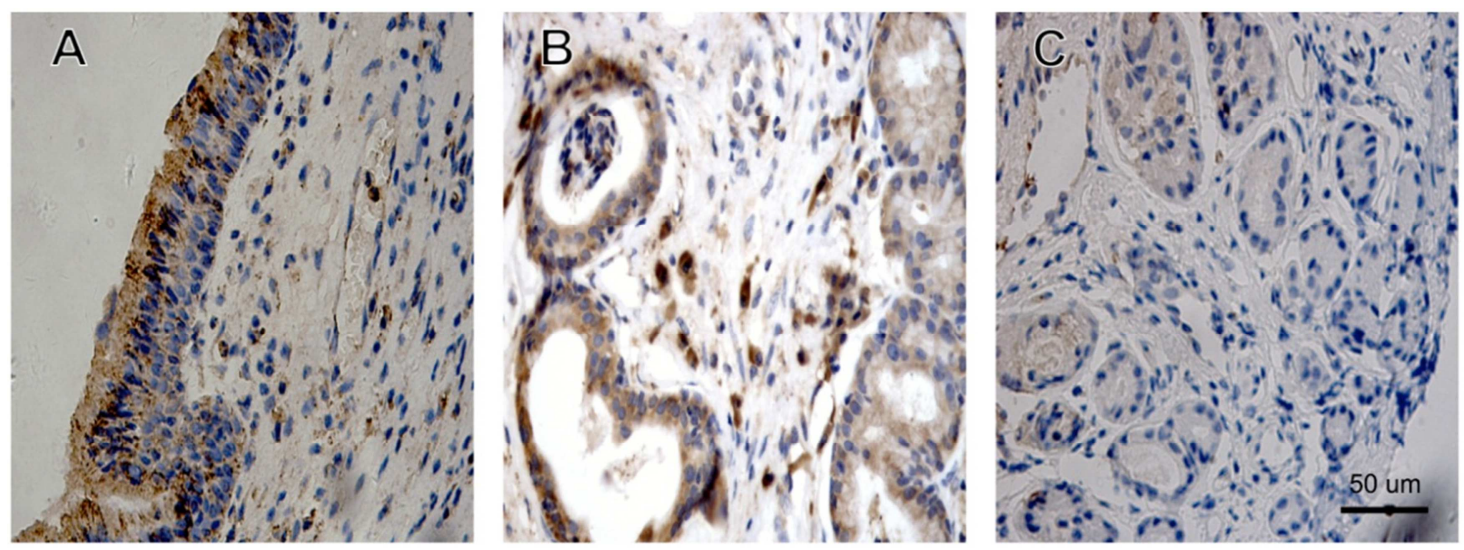

Figure 2. The expression of VEGF. (A) VEGF in mucosal epithelium of nasal polyps. (B) VEGF in glandular epithelium of nasal polyps. (C) VEGF in inferior turbinate tissue (low expression). 
CD34 in nasal polyp showed microvascular morphology (Figure 3): the expression of CD34 was mainly in cytoplasmic, with weak staining in inferior turbinate tissue. The morphology of microvascular was irregular, round or

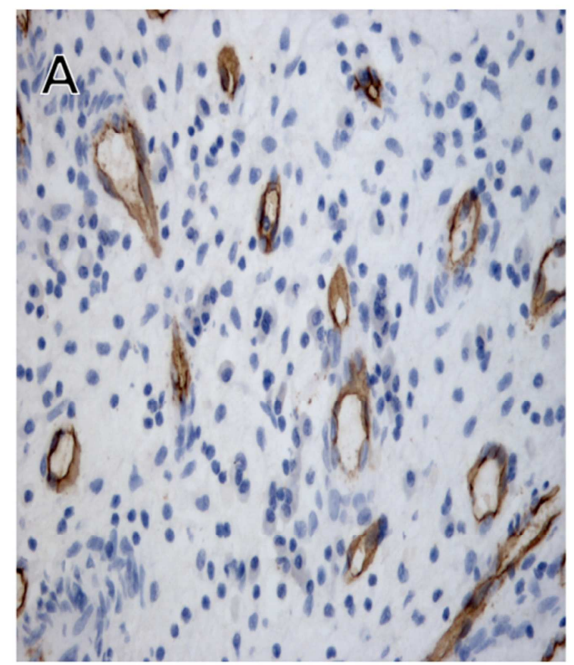

cord shaped, mostly CD34 positive staining. The MVD values can be found in Table 1, the scores was $22.67 \pm 7.54$ in nasal polyp tissues and $7.04 \pm 2.63$ in inferior turbinate tissues, showing significant difference $(t=16.9, P<0.01)$.

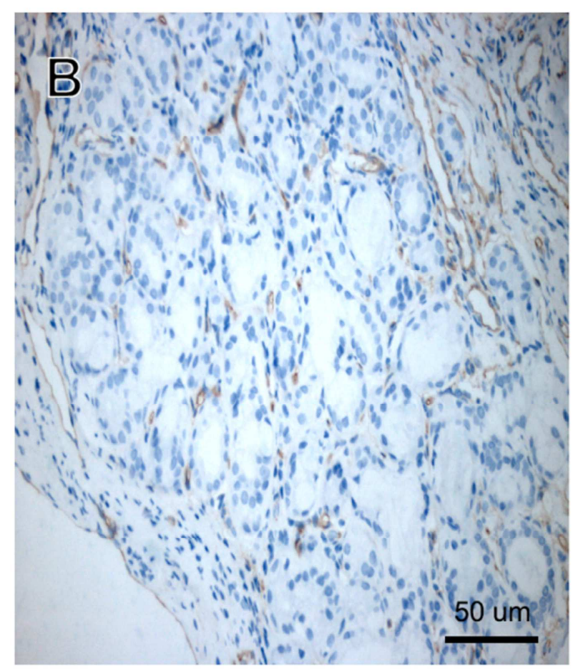

Figure 3. The expression of CD34. (A) CD34 in mucosal epithelium of nasal polyps. (B) CD34 in in inferior turbinate tissue.

Table 1. MVD score and expressions of STAT6 and VEGF in the nasal polyps and the inferior turbinate.

\begin{tabular}{lllll}
\hline Group & Case & STAT6 (case, \%) & VEGF (case, \%) & MVD \\
\hline Nasal polyps & 40 & $28(70 \%)$ & $30(75 \%)$ & $22.67 \pm 7.54$ \\
Inferior turbinate & 10 & 0 & $1(10 \%)$ & $7.04 \pm 2.63$ \\
\hline
\end{tabular}

Then we analyzed the correlation between the expression of STAT6 and VEGF in nasal polyps. Staining of STAT6 was divided into 4 levels: $(-),(+),(++),(+++)$. Staining of VEGF was divided into 4 levels: $0,1,2,3$. According to KruskalWallis Test, the correlation was analyzed and showed in Table 2 .

Table 2. The correlation between the expression of STAT6 and VEGF in the nasal polyps.

\begin{tabular}{llllll}
\hline \multirow{2}{*}{ VEGF } & \multirow{2}{*}{ Total } & \multicolumn{2}{c}{ STAT6 } & & ++ \\
\cline { 3 - 6 } & & - & + & ++ \\
\hline 0 & 11 & 5 & 3 & 2 & 1 \\
1 & 8 & 2 & 3 & 2 & 1 \\
2 & 12 & 1 & 3 & 5 & 3 \\
3 & 9 & 1 & 2 & 3 & 3 \\
Total & 40 & 9 & 11 & 12 & 8 \\
\hline
\end{tabular}

$\chi^{2}=11.3, P=0.038$

\section{Discussion}

STAT6 was firstly identified in IFN induced gene transcription. There are 5 important functional regions located in STAT6: (1) C-terminal transcriptional activation region; (2) Tyr641 is important for IL-4 induced STAT6 activation; (3) highly conserved SH2 domain, where IL-4 receptor binding to and the region for STAT6 dimers; (4) DNA binding domain; (5) conserved $\mathrm{N}$ terminal region [9]. Human STAT6 located in 12q13-24 chromosomal region, is one of the most potential candidate for the treatment of allergic rhinitis and asthma [10]. Via the JAK/STAT pathway, STAT6 regulates the expression of IL-3, IL-4 and IL-13, which plays an important role in inflammatory response [11, 12], involving formation of inflammatory vessels, cell proliferation, cell cycle and apoptosis. In the current study, we found the high expression of STAT6 in nasal polyps and glandular epitheliums. Thus, STAT6 may be critical for cell proliferation and apoptosis inhibition of nasal polyps.

Two mechanisms were believed to be involved in VEGF induced vascular formation: (1) as an endothelial cell mitogen; (2) as a potent regulator of vascular permeability. Under normal physiological conditions, angiogenesis is shut down, with lower VEGF expressed [13]. With the increased VEGF expression, vascular permeability is increasing, the invasion of inflammatory cells, fibroblasts and endothelium cells would induce the formation of granulation tissue. Meanwhile, because of increased plasma extravasation, extracellular matrix accumulation and tissue edema, local increased force induces epithelial rupture, slowly forming nasal polyps $[14,15]$. In the current study, the positive expression of VEGF in nasal polyps was $75 \%$, while low expression in inferior turbinate tissue, indicating the important role of VEGF in the development of nasal polyps. This is consistent with previous reports $[16,17]$. CD34 is expressed in the inner surface of vascular endothelium cells, indicating the involvement of CD34 antigen in endothelium adhesion and migration during the vascular formation. In the current study, CD34 was positively expressed in nasal polyps; and the MVD score was markedly higher than that in 
control group, suggesting obvious vascular hyperplasia in nasal polyps. Accordingly, vascular formation is important for the formation, development and recurrence process of nasal polyps $[14,17]$. Inducing neovascularization can promote the formation of nasal polyps, which involved many cytokines; and VEGF may be a critical one. Further, the genetic expression of VEGF may be regulated by STAT proteins [18]. STATs may function at the upstream of VEGF to control its expression, which need further evidences.

In summary, we determined the relationship of STAT6, VEGF and MVD score in nasal polyps, provided evidence for the interaction between them in clinical specimens, indicating the important roles in the development of nasal polyps. However, the detailed mechanisms involving STAT6, VEGF and MVD or other cytokines need to be further explored.

\section{Acknowledgements}

The authors declare no conflict of interest. This work was supported by Medical Scientific Research Foundation of Guangdong Province, China (A2017238).

\section{References}

[1] Sivasubramaniam, R. and R. J. Harvey, How to Assess, Control, and Manage Uncontrolled CRS/Nasal Polyp Patients. Curr Allergy Asthma Rep, 2017. 17(9): p. 58.

[2] Hsu, J. and A. T. Peters, Pathophysiology of chronic rhinosinusitis with nasal polyp. Am J Rhinol Allergy, 2011. 25(5): p. 285-90.

[3] Goenka, S. and M. H. Kaplan, Transcriptional regulation by STAT6. Immunol Res, 2011. 50(1): p. 87-96.

[4] Yamaoka, K., K. Maeshima, S. Kubo, and Y. Tanaka, [Regulation of inflammation through JAK3-Stat6 pathway in dendritic cells]. Nihon Rinsho Meneki Gakkai Kaishi, 2012. 35(1): p. $62-8$.

[5] Li, B. H., S. B. Xu, F. Li, X. G. Zou, A. Saimaiti, D. Simayi, Y. H. Wang, Y. Zhang, J. Yuan, and W. J. Zhang, Stat6 activity-related Th2 cytokine profile and tumor growth advantage of human colorectal cancer cells in vitro and in vivo. Cell Signal, 2012. 24(3): p. 718-25.

[6] Zafirellis, K., G. Agrogiannis, A. Zachaki, K. Gravani, A. Karameris, and C. Kombouras, Prognostic significance of $V E G F$ expression evaluated by quantitative immunohistochemical analysis in colorectal cancer. J Surg Res, 2008. 147(1): p. 99-107.
[7] Slomka, M., O. Stasikowska, M. Wagrowska-Danilewicz, M. Danilewicz, and E. Malecka-Panas, [Diagnostic and prognostic values of repair protein $h M L H 1, h M S H 2$ and protein CD34 immunoexpression in sporadic colorectal cancer]. Pol Merkur Lekarski, 2010. 29(174): p. 351-6.

[8] Kimura, H., T. Nakajima, K. Kagawa, T. Deguchi, M. Kakusui, T. Katagishi, T. Okanoue, K. Kashima, and T. Ashihara, Angiogenesis in hepatocellular carcinoma as evaluated by CD34 immunohistochemistry. Liver, 1998. 18(1): p. 14-9.

[9] Kisseleva, T., S. Bhattacharya, J. Braunstein, and C. W. Schindler, Signaling through the JAK/STAT pathway, recent advances and future challenges. Gene, 2002. 285(1-2): p. 124.

[10] Wurster, A. L., T. Tanaka, and M. J. Grusby, The biology of Stat4 and Stat6. Oncogene, 2000. 19(21): p. 2577-84.

[11] Lau, M., E. Tsantikos, M. J. Maxwell, D. M. Tarlinton, G. P. Anderson, and M. L. Hibbs, Loss of STAT6 promotes autoimmune disease and atopy on a susceptible genetic background. J Autoimmun, 2012. 39(4): p. 388-97.

[12] Maier, E., A. Duschl, and J. Horejs-Hoeck, STAT6-dependent and -independent mechanisms in Th2 polarization. Eur $\mathrm{J}$ Immunol, 2012. 42(11): p. 2827-33.

[13] Iyer, S. and K. R. Acharya, Tying the knot: the cystine signature and molecular-recognition processes of the vascular endothelial growth factor family of angiogenic cytokines. FEBS J, 2011. 278(22): p. 4304-22.

[14] Muluk, N. B., P. Atasoy, O. K. Arikan, and C. Koc, Role of vascular endothelial growth factor in the pathogenesis of nasal polyps. J Otolaryngol, 2007. 36(6): p. 357-66.

[15] Park, S. K., H I. Kim, Y. I. Yang, and D. Y. Hur, Effects of methotrexate on vascular endothelial growth factor, angiopoietin 1, and angiopoietin 2 in nasal polyps. Am J Rhinol Allergy, 2011. 25(4): p. e129-32.

[16] Wittekindt, C., A. Hess, W. Bloch, S. Sultanie, and O. Michel, Immunohistochemical expression of VEGF and VEGF receptors in nasal polyps as compared to normal turbinate muсоsa. Eur Arch Otorhinolaryngol, 2002. 259(6): p. 294-8.

[17] Lee, H. S., A. Myers, and J. Kim, Vascular endothelial growth factor drives autocrine epithelial cell proliferation and survival in chronic rhinosinusitis with nasal polyposis. Am J Respir Crit Care Med, 2009. 180(11): p. 1056-67.

[18] Chen, Z. and Z. C. Han, STAT3: a critical transcription activator in angiogenesis. Med Res Rev, 2008. 28(2): p. 185200 . 